\title{
Evaluation of storage energy of the constructional steel during plastic deformation
}

\author{
by A.M. Ivanov*, E.S. Lukin*, and B.G. Vainer** \\ * Institute of Physical and Technical Problems of the North, Yakutsk, Russia \\ ** Institute of Semiconductor Physics, Novosibirsk, Russia
}

\begin{abstract}
The results concerned with the evaluation of storage energy in the process of plastic deformation of the constructional steel are presented. Temperature measurements during the static tensile tests were conducted using an FPA-based infrared camera. The heat evolved in the process of plastic deformation was determined by solving the heat conduction equation simultaneously with the use of infrared radiation measurement data. The obtained data of energy storage within the range of rates from $9 \times 10^{-4} \mathrm{~s}^{-1}$ to $9 \times 10^{-3} \mathrm{~s}^{-1}$ do not depend appreciably on deformation rate.
\end{abstract}

\section{Introduction}

Generally, plastic deformation of the constructional steel is accompanied with the generation and motion of dislocations. Change of the density and configuration of the defects always leads to energy dissipation, which causes the specimen temperature increase. The phenomenon of temperature increase in the process of plastic deformation of solids is known as thermoplastic effect.

The part of work of plastic deformation $A_{P}$ is absorbed by a material while the another part is transformed into released heat $Q$. Thus the storage energy $E_{S}$ is determined as a difference between the work of plastic deformation and the heat released into surroundings [1]:

$$
d E_{S}=d A_{P}-d Q
$$

Work of plastic deformation is usually determined from the stress-strain curve. The heat $Q$ can be evaluated with the use of calorimeter [2, 3]. However, the calorimetric method is limited by its low speed of response [3].

Besides, the investigations of deformation in metals and steels are sometimes carried out with the use of infrared imaging cameras [4 - 6]. In this case, the change in sample temperature during deformation is measured. However, the correct determination of the released heat during deformation remains the difficult problem and needs to be studied. In the latter case, the heat $Q$ can be determined by simulation of the process of sample heating during deformation by applying electrical power $P(t)$ in such a way that the temperature increase with time $t$ during the simulation is identical with that measured during tensile testing [5].

\section{The materials and instruments tested}

The goal of the present work is to evaluate the energy storage in the process of plastic deformation of the constructional steel. The standard sheet specimens from the steel $18 \mathrm{G} 2 \mathrm{~S}$ were subjected to static tensile test at various rates 
of deformation: $9 \times 10^{-4} \mathrm{~s}^{-1}, 2 \times 10^{-3} \mathrm{~s}^{-1}, 4 \times 10^{-3} \mathrm{~s}^{-1}$, and $9 \times 10^{-3} \mathrm{~s}^{-1}$. The chemical composition of the steel was 0.15 wt $\% \mathrm{C}, 0.85 \mathrm{wt} \% \mathrm{Si}, 1.4 \mathrm{wt} \% \mathrm{Mn}, 0.25 \mathrm{wt} \% \mathrm{Cr}$, $0.15 w t \% \mathrm{Ni}$, and $0.31 \mathrm{wt} \% \mathrm{Cu}$. Dimensions of the tested specimens were $0.075 \mathrm{~m}$ - length, $0.02 \mathrm{~m}$ - width, and $0.002 \mathrm{~m}$ - thickness. Tensile tests were performed on the "Instron-1195" testing machine with a constant strain rate. The yield strength and the ultimate strength of steel $18 \mathrm{G} 2 \mathrm{~S}$ were $410 \mathrm{MPa}$ and $560 \mathrm{MPa}$, respectively.

Temperature measurements during the specimen deformation were performed using the infrared thermography system TKVr-IFP/"SVIT" equipped with a computer. The temperature sensitivity of the infrared camera (NETD at $30{ }^{\circ} \mathrm{C}$ ) was $0.028{ }^{\circ} \mathrm{C}$. The field of view was about $0.09 \mathrm{~m} \times 0.09 \mathrm{~m}$ at the distance between the camera and specimen of $0.3 \mathrm{~m}$. IR images were stored in the computer memory at frequency 20 frames per second. The sensitivity spectral range of the device was $2.45 \div 3.05 \mu \mathrm{m}$. The analysis of both surface temperature map and stress diagram was carried out to evaluate the energy storage during plastic deformation.

\section{Work of plastic deformation}

When material is deformed, a certain fraction of total work $A$ is expended on the work of elastic deformation $A_{e}$ and another one - on the work of plastic deformation $A_{P}$. From this it follows that

$$
A_{P}=A-A_{e}
$$

Principle of determining the work of plastic deformation $A_{P}$ during the tensile test is presented in Figure 1, where $\sigma$-stress, $\varepsilon$ - strain. Total work $A$ is determined as the shaded area between the stress-strain curve 1 and the straight line 2 . Work of elastic deformation $A_{e}$ is shown as a twice shaded area between the straight lines 2 and 3.

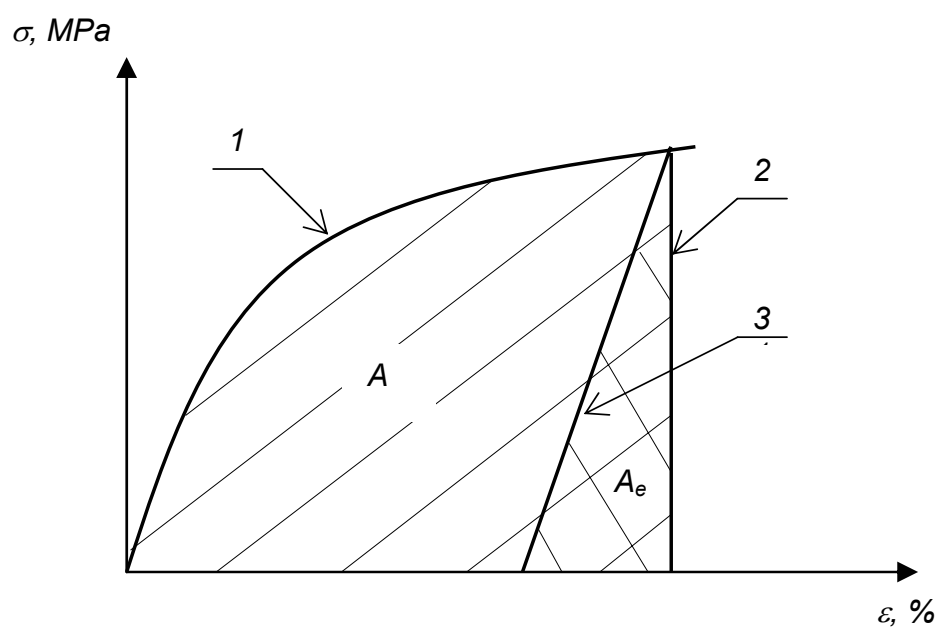

Fig. 1. Principle of determination of the work of plastic deformation during tensile test 
The dependence of specific work of plastic deformation $a_{p}=A_{P} / V(V-$ the volume of a specimen) on relative elongation $\varepsilon \%$ at different strain rates is presented in Figure 2. As it follows from Figure 2, the difference between works of plastic deformation made at different strain rates does not exceed $4 \%$.

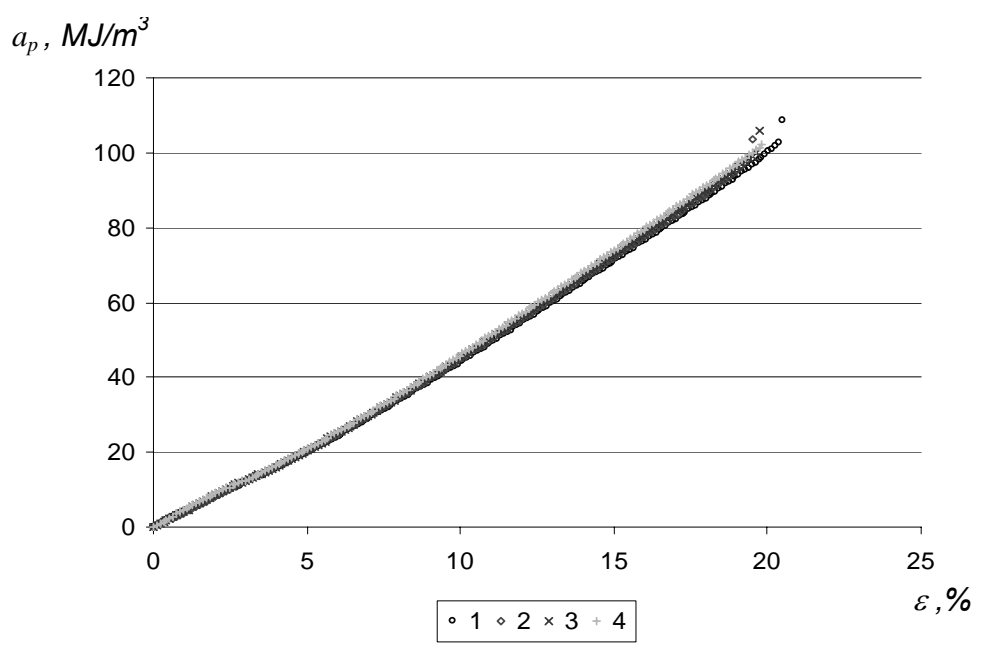

Fig. 2. The curves of plastic deformation specific work of steel $18 \Gamma 2 \mathrm{C}$ at different strain rates $\dot{\varepsilon}$ vs. relative elongation : 1 - at $\dot{\varepsilon}=8,98 \times 10^{-4} \mathrm{~s}^{-1} ; 2-$ $\dot{\varepsilon}=1,78 \times 10^{-3} \mathrm{~s}^{-1} ; 3-\dot{\varepsilon}=3,57 \times 10^{-3} \mathrm{~s}^{-1} ; 4-\dot{\varepsilon}=8,93 \times 10^{-3} \mathrm{~s}^{-1}$

\section{Evaluation of the evolved heat}

In this paper, the heat evolved in the process of plastic deformation is determined by solving the heat conduction equation simultaneously with the use of infrared radiation measurement data.

The evolved heat $Q$ during deformation is evaluated from the solution of one-dimensional heat conduction equation with a heat source $q(t)$ :

$$
\frac{\partial T(x, t)}{\partial t}=a \frac{\partial^{2} T(x, t)}{\partial x^{2}}-v\left(T(x, t)-T_{c}\right)+\frac{q(t)}{c \rho}
$$

Here, $a$ is the temperature conductivity, $v$ - thermal diffusivity, $c$ - heat capacity, $\rho$ - density, $T(x, t)$ and $T_{c}$ are the distribution of temperature on surface of the specimen and the temperature of surroundings, respectively. Within this problem, the specimen was simulated as an axisymmetric finite-size bar with known boundary conditions: 


$$
T(x, 0)=T_{c}, \quad T(0, t)=T_{c}, \quad T(l, t)=T_{c} .
$$

Solutions of the heat conduction equation (3) for the heat source are:

$$
q(t)=\frac{\Delta \bar{T}(x, t)}{a_{1}-a_{2} \cdot \exp \left[-b_{1} \cdot t\right]-a_{3} \cdot \exp \left[-b_{2} \cdot t\right]}
$$

where $a_{1}, a_{2}, a_{3}, b_{1}$, and $b_{2}$ are the constants dependent on the length of bar, thermal conductivity, density, and heat capacity. The temperature distribution $\Delta \bar{T}(x, t)$ is obtained empirically with the use of infrared system. Dependence of the heat source power on time is presented in Figure 3. The evolved heat $q$ (Eq. (6)) can be obtained from the integration of solutions (5) of heat conduction:

$$
q=\int_{0}^{t} q(t) \cdot d t
$$

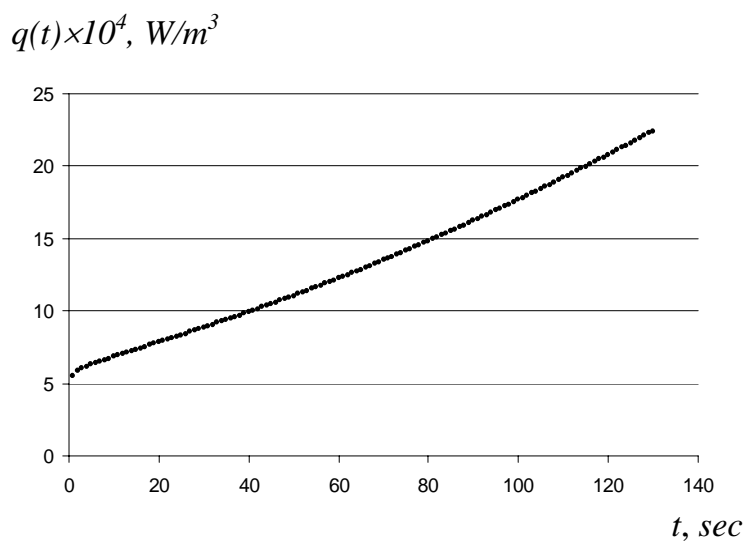

Fig. 3. Dependence of the heat source power of steel $18 \mathrm{G} 2 \mathrm{~S}$ on time

\section{Results}

The dependences of the specific work of plastic deformation $a_{P}$, specific storage energy $e_{S}$ and evolved heat $q$ in the tensile tests on strain of the steel 18G2S are presented in Figure 4. It is seen that the amount of evolved heat makes up to $30 \%$ of work of plastic deformation, and the rest of this work is stored by material. The obtained results differ from the data presented in [5] where the amount of evolved heat for the stainless steel makes $60-70 \%$ of the work of plastic deformation. At the same time, the absolute value of the storage energy for 18G2S steel exceeds that for stainless steel and amounts to $37.5 \mathrm{MJ} / \mathrm{m}^{3}$ and $30 \mathrm{MJ} / \mathrm{m}^{3}$, respectively. 
Dependence of the storage energy at different deformation rates on strain is shown in Figure 5. The difference between storage energies amounts to not more than $12 \%$. One can conclude that the storage energy within the range of rates considered above does not depend appreciably on deformation rate.

$e, M J / m^{3}$

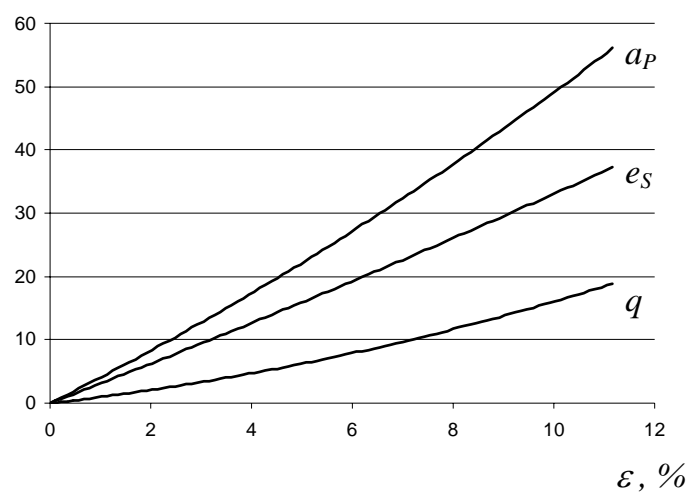

Fig. 4. Dependence of the specific plastic work $a_{P}$, specific storage energy $e_{S}$ and evolved heat $q$ in the tensile on strain of the steel 18G2S.

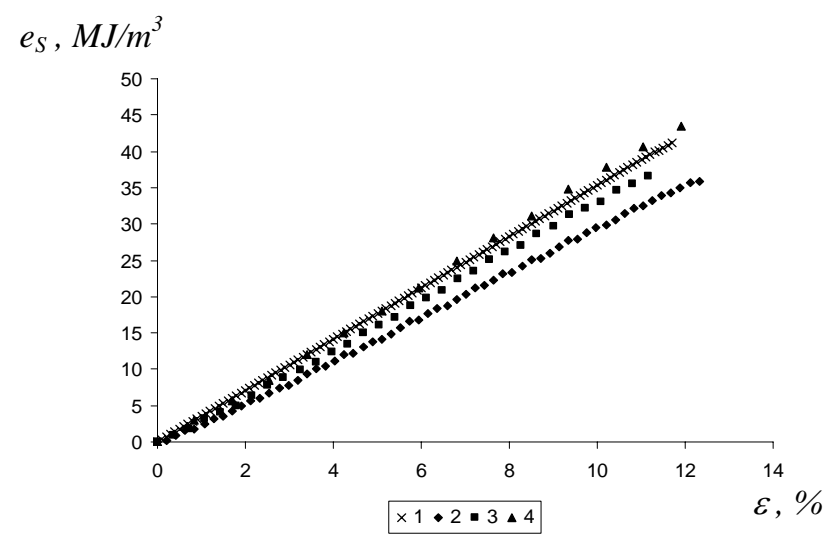

Fig. 5. Dependence of the specific storage energy received by the different deformation rates on strain, where $1-$ by $\dot{\varepsilon}=8,98 \times 10^{-4} \mathrm{~s}^{-1} ; 2-\dot{\varepsilon}=$ $1,78 \times 10^{-3} \mathrm{~s}^{-1} ; 3-\dot{\varepsilon}=3,57 \times 10^{-3} \mathrm{~s}^{-1} ; 4-\dot{\varepsilon}=8,93 \times 10^{-3} \mathrm{~s}^{-1}$. 
Thus, solution of the inverse problem of conductivity allows to evaluate the amount of heat evolved under the influence of thermoplastic effect with taking into account the heat dissipated into surroundings. The described above calculationexperiential technique exploiting infrared imaging camera enables to determine the energy stored by the material at static tensile test of specimens.

\section{Acknowledgements}

This work has been supported by the Russian Foundation for Basic Research under Grant RFBR No. 06-01-96007.

\section{REFERENCES}

[1] Taylor G.I., Quinney H. The latent energy remaining in a metal after cold working. //Proc. Roy. Soc., 1934, vol. CXLIII.-A., P. 307-326.

[2] Maksimkin O.P., Gusev M.N. Some peculiarities of energy dissipation during plastic deformation of iron and niobium. // Pis'ma v Zhurnal Tekhnicheskoi Fiziki, 2001. - V. 27. - No.24. - P. 85-89.

[3] Astafiev I.V., Maksimkin O.P. Restoration of calorimetric thermogramms in the experiments on heat emission and energy storing during deformation. // Industrial laboratory. - 1994. - No.1. - P. 44-46.

[4] Oliferuk W. Investigation of metal deformation using thermography. // Quantitative infrared thermography 4, QIRT'98, Proceedings of Eurotherm Seminar No. 60. - Lodz, Poland, 2000. - P. 134-139.

[5] Pieczyska E.A., Gadaj S.P., Nowacki W.K. Rate of energy storage during consecutive deformation of steel. // Quantitative infrared thermography 5, QIRT'2000, Proceedings of Eurotherm Seminar No. 64. - Reims, France, 2000. - P. 260-264.

[6] Lukin E.S., Ivanov A.M., Vainer B.G. Thermal imaging investigations in experimental mechanics. // Russian Journal of Nondestructive Testing, 2003. - V. 39. - No. 6. - P. 472-477. 